RESEARCH

Turkish Journal of Geriatrics

DOI: 10.31086/tigeri.2020.154

2020; 23(2): 197-205

- Sinan $\mathrm{AVCl}^{1}$ (D)

- Volkan ÇAĞLAYAN ${ }^{1}$ D

. Efe ÖNEN ${ }^{1}$ iD

- Metin KILIÇ' ${ }^{1 D}$

- Abdullah GÜL ${ }^{1}$ (D)

- Sedat ÖNER'

CORRESPONDANCE

AvcI SINAN

University of Health Sciences, Bursa Yuksek

Intisas Training and Research Hospital, Urology

Clinic, Bursa, TURKEY.

Phone: +905063375824

e-mail: sinavci@yahoo.com

Received: April 06, 2020

Accepted: May 03, 2020

${ }^{1}$ University of Health Sciences, Bursa Yuksek Ihtisas Training and Research Hospital, Urology Clinic, Bursa, TURKEY

\section{THE EFFECTIVENESS OF PROSTATE SPECIFIC ANTIGEN DENSITY IN PREDICTING PROSTATE CANCER AND CLINICALLY SIGNIFICANT PROSTATE CANCER IN PATIENTS AGED 70 YEARS AND OVER}

\section{Abstract}

Objectives: To evaluate the effects of free-to-total prostate specific antigen ratio, prostate specific antigen density and prostate volume in predicting prostate cancer and clinically significant cancer in patients aged 70 years or older and in establishing eligible cut-off levels.

Materials and Method: A retrospective study was done on the data of 1848 men who underwent prostate biopsy in our clinic between January 2015 and October 2019. Patients with prostate specific antigen levels $>10 \mathrm{ng} / \mathrm{mL}$ were excluded. Volumes were obtained by transrectal ultrasound. A clinically significant cancer was defined as having a Gleason score of $\geq 7$.

Results: 130 patients with available data were enrolled in the study. Prostate specific antigen density, prostate volume and free-to-total prostate specific antigen ratio were significantly different in patients with or without cancer. Regarding area under curve, prostate specific antigen density and prostate volume showed similar efficacy, and both performed better than free-to-total prostate specific antigen ratio (Area under curves for prostate specific antigen density, prostate volume and freeto-total prostate specific antigen ratio were $=0.770,0.769$ and 0.627 , respectively). A multivariate analysis showed that only prostate specific antigen density was an independent predictor for prostate cancer and clinically significant prostate cancer, with cut-off values of 0.11 and 0.199 , respectively.

Conclusion: Prostate specific antigen density is seen as one step ahead of prostate volume and free-to-total prostate specific antigen ratio in predicting prostate cancer and clinically significant prostate cancer. Prostate specific antigen density may play an active role in deciding prostate biopsies to prevent unnecessary tests in elderly.

Key Words: Geriatrics; Prostatic Neoplasms; Prostate-Specific Antigen. 


\section{INTRODUCTION}

Prostate cancer ( $\mathrm{PCa}$ ) is the second most commonly diagnosed cancer and the second leading cause of cancer-related death in men worldwide, with an estimated 1.1 million diagnoses in 2012, accounting for $15 \%$ of all diagnosed cancers $(1,2)$. The prostate-specific antigen (PSA) is widely used for early disease detection. However, though highly sensitive, PSA is less specific for the diagnosis of $\mathrm{PCa}$, resulting in difficulties in differentiating malignant and benign prostatic conditions in men. Hence, several investigations have been done to improve cancer detection rates using various parameters including free-to-total PSA ratio ( $f$ / tPSA), PSA density (PSAD), PSA velocity and age-referenced PSA. These prediction methods developed to reduce unnecessary biopsies and to avoid false-negative biopsies, are more accurate than PSA screening alone (3). Contrarily, majority of these studies were conducted in patients aged 40-70 years. Thus, there is a limited discussion of biopsy strategy in older, referral populations with symptoms in whom the incidence of latent cancer is relatively high and age-related benign PSA elevation is common (4). Whether sole PSA, PSA-associated parameters ( $f / t P S A, P S A D)$ and prostate volume (PV) are useful tools in distinguishing PCa from benign prostatic disease in older populations, similar to the situation in younger ones, has yet to be determined.

Data from the Goteborg arm of the ERSPC trial (5) noted that the age at which early diagnosis should be stopped remains controversial, and in parallel to this situation, there is no upper age limit to perform prostate biopsy in the European Association of Urology (EAU) guidelines. Nevertheless, EAU guidelines recommend an individualised risk-adapted biopsy strategy for early detection to a well-informed man in combination with patients' wishes and at least 1015 years of life expectancy. It is evident that there is an increasing proportion of individuals aged 70 years and older and an increasing life expectancy worldwide (6). As a result, urologists are faced with healthier patients aged 70 years and older who are candidates for prostate biopsy. However, performing prostate biopsies in men older than 70 years is associated with a nearly fourfold increased risk of complications and longer hospitalisations (7). Thus, managing prostate biopsy in the elderly is of increasing importance.

In an effort to contribute to the existing limited data on biopsy strategy in patients 70 years or older, this study aimed to evaluate the effectiveness of PSAD, PV and f/tPSA in predicting PCa in patients of this age group, in which PSA is likely to be elevated due to above-mentioned reasons, to avoid unnecessary biopsies. In addition, since the main goal of trying to predict PCa is actually to predict clinically significant $\mathrm{PC}$, we also evaluated the parameters to predict clinically significant PCa with a Gleason score of 7 or above. Finally, it was aimed to determine the appropriate cutting values, from which we can make these separations.

\section{MATERIALS AND METHOD}

A retrospective analysis was done on the data of 1848 men who underwent transrectal ultrasound (TRUS)-guided prostate biopsy in our clinic between January 2015 and October 2019. Biopsy decisions were taken in a case-by-case basis, considering the patients' digital rectal examination (DRE) findings, PSA values according to age, comorbidities and life expectancies. Patients with PSA levels $>10 \mathrm{ng} / \mathrm{mL}$, aged $<70$ years and with a history of 5alpha-reductase inhibitor therapy, phytotherapy or any invasive therapy for benign prostate hyperplasia (BPH) were excluded. Patients who had a cystoscopy, colonoscopy, TRUS, acute prostatitis, urinary tract infection and urinary retention a month prior to the study were also eliminated.

Serum PSA levels were measured in $\mathrm{ng} /$ $\mathrm{mL}$ using the chemiluminescent microparticle immunoassay (CMIA) and all serum samples were 
drawn before any prostate manipulation such as DRE, TRUS and biopsy. f/tPSA was calculated as the ratio of free PSA to total PSA multiplied by 100. The pre-biopsy PV of the patients were obtained by measuring three dimensions of the prostate with TRUS and using the ellipsoid formula (PV= height $\times$ width $\times$ length $\times 0.52$ ). Patients who have received at least 12 -core biopsies were included in the study. Histological evaluation identified each patient's prostate specimen into either cancerous (prostate adenocarcinoma) or non-cancerous (Benign prostatic hyperplasia (BPH) and/or chronic prostatitis). Clinically significant PCa was defined as having a Gleason score $\geq 7$.

The patients' age, total-free PSA value, pathology results and Gleason score were evaluated, and the impacts of PSAD, PV, and $f / t P S A$ on the detection of PCa and clinically significant $\mathrm{PC}$ a were investigated.

\section{Statistical Analysis}

Data obtained in the study were analysed using SPSS version 15.0 software (SPSS, Inc., Chicago, IL, USA). The conformance of the data to the normal distribution curve was evaluated using the Shapiro-Wilk test, and the continuous and categorical data were compared using the MannWhitney U-test, the chi-square test and KruskalWallis test, respectively. The relationship of biopsy results with age, total-free PSA, PV, f/tPSA, PSAD and the Gleason score was investigated through a univariate analysis using the Mann-Whitney $U$ and chi-square tests. A logistic regression multivariate analysis was done to determine independent predictive factors for malignant prostate biopsy results. The receiver operating characteristic (ROC) curve was applied to evaluate and compare the efficacy of PV, PSAD and f/tPSA for PCa diagnosis. A $p$-value of $<0.05$ was considered statistically significant.

\section{RESULTS}

After applying the exclusion criteria, 130 patients with available data of age, total-free PSA levels and PV calculated by TRUS were enrolled in the study. PCa and BPH were detected in 40 patients (30.8\%) and in 90 patients (69.2\%), respectively. Table 1 shows the clinical characteristics of patients.

On univariate analysis, f/tPSA and PV was significantly lower and PSAD was significantly higher in PCa patients than in patients with benign prostatic condition, whereas PSA, free PSA and age showed no significant difference between the two groups. In ROC analysis, the order of AUCs was determined as PSAD > PV > f/tPSA (Table 2).

A multivariate analysis was carried out to compare PSAD, PV and f/tPSA which were significant in a univariate analysis. The multivariate

Table 1. The clinical characteristics of patients.

\begin{tabular}{|c|c|c|c|c|c|c|c|c|c|}
\hline & \multirow{2}{*}{ Age (years) } & \multirow{2}{*}{$\begin{array}{r}\text { total PSA } \\
(\mathrm{ng} / \mathrm{mL})\end{array}$} & \multirow{2}{*}{$\begin{array}{r}\text { free PSA } \\
(\mathrm{ng} / \mathrm{mL})\end{array}$} & \multirow{2}{*}{$\begin{array}{l}\text { free/total } \\
\text { PSA ratio }\end{array}$} & \multirow{2}{*}{$\begin{array}{l}\text { PV } \\
\text { (cc) }\end{array}$} & \multirow{2}{*}{$\begin{array}{r}\text { PSAD (ng/ } \\
\mathrm{mL} / \mathrm{cc}\end{array}$} & \multicolumn{3}{|c|}{ Biopsy results } \\
\hline & & & & & & & $\mathrm{PCa}$ & BPH & Total \\
\hline Mean & 73.77 & 7.109 & 1.822 & 0.261 & 72.73 & 0.129 & & & \\
\hline & \pm & & & & & & - & - & - \\
\hline SD & 2.95 & 1.759 & 0.886 & 0.115 & 33.78 & 0.092 & & & \\
\hline Median & 73 & 7.085 & 1.695 & 0.247 & 71 & 0.101 & & & \\
\hline (IQR) & $(71-76)$ & $(5.845-8.550)$ & $(1.21-2.21)$ & $(0.178-0.324)$ & $(46.25-94.25)$ & $(0.070-0.144)$ & - & - & \\
\hline$n(\%)$ & & & & & & & $\begin{array}{r}40 \\
(30.8)\end{array}$ & $\begin{array}{r}90 \\
(69.2)\end{array}$ & $\begin{array}{r}130 \\
(100)\end{array}$ \\
\hline
\end{tabular}

PV: prostate volume, PSA: prostate specific antigen, PSAD: prostate specific antigen density, PCa: prostate cancer, BPH: benign prostate hyperplasia, SD: standard deviation, IQR: interquartile range. 
Table 2. Comparison of patients' age, total PSA, free PSA, free to total PSA ratio, PSA density and prostate volume between cancer and non-cancer groups.

\begin{tabular}{|c|c|c|c|c|}
\hline \multirow[b]{2}{*}{ Parameters } & \multicolumn{2}{|r|}{ Biopsy result } & \multirow[b]{2}{*}{$p$ value } & \multirow[b]{2}{*}{ AUC } \\
\hline & Cancer & Non-cancer & & \\
\hline $\begin{array}{r}\text { Number of patients } \\
n(\%)\end{array}$ & $\begin{array}{r}40 \\
(30.8)\end{array}$ & $\begin{array}{r}90 \\
(69.2)\end{array}$ & & \\
\hline $\begin{array}{r}\text { Age (years) } \\
\text { Mean } \pm S D \\
\text { Median (IQR) }\end{array}$ & $\begin{array}{r}73.781 \pm 2.684 \\
73(72-75.75)\end{array}$ & $\begin{array}{r}73.77 \pm 3.80 \\
73(71-76)\end{array}$ & 0.782 & 0.548 \\
\hline $\begin{array}{r}\text { Total PSA }(\mathrm{ng} / \mathrm{mL}) \\
\text { Mean } \pm \mathrm{SD} \\
\text { Median }(\mathrm{IQR}) \\
\end{array}$ & $\begin{array}{r}7.47 \pm 1.725 \\
7.75(6.44-8.79)\end{array}$ & $\begin{array}{r}6.945 \pm 1.758 \\
6.91(5.58-8.42)\end{array}$ & 0.093 & 0.639 \\
\hline $\begin{array}{r}\text { Free PSA }(\mathrm{ng} / \mathrm{mL}) \\
\text { Mean } \pm S D \\
\text { Median }(\mathrm{IQR}) \\
\end{array}$ & $\begin{array}{r}1.805 \pm 1.234 \\
1.46(1.14-2.12)\end{array}$ & $\begin{array}{r}1.829 \pm 0.687 \\
1.795(1.297-2.335)\end{array}$ & 0.124 & 0.630 \\
\hline $\begin{array}{r}\text { Free/Total PSA ratio } \\
\text { Mean } \pm \text { SD } \\
\text { Median (IQR) } \\
\end{array}$ & $\begin{array}{r}0.242 \pm 0.148 \\
0.214(0.146-0.289)\end{array}$ & $\begin{array}{r}0.270 \pm 0.096 \\
0.256(0.187-0.338)\end{array}$ & $<0,021$ & 0.627 \\
\hline $\begin{array}{r}\mathrm{PV}(\mathrm{cc}) \\
\text { Mean } \pm \mathrm{SD} \\
\text { Median (IOR) } \\
\end{array}$ & $\begin{array}{r}52.28 \pm 29.01 \\
47(30.5-67.25)\end{array}$ & $\begin{array}{l}81.82 \pm 31.835 \\
82(60.25-100)\end{array}$ & $<0,001$ & 0.769 \\
\hline $\begin{array}{r}\text { PSDA (ng/mL/cc) } \\
\text { Mean } \pm S D \\
\text { Median (IOR) }\end{array}$ & $\begin{array}{r}0.189 \pm 0.109 \\
0.157(0.099-0.275)\end{array}$ & $\begin{array}{r}0.102 \pm 0.068 \\
0.085(0.066-0.118)\end{array}$ & $<0,001$ & 0.770 \\
\hline
\end{tabular}

PV: prostate volume, PSA: prostate specific antigen, PSAD: prostate specific antigen density, AUC: area under curve, SD: standard deviation, IQR: interquartile range.

analysis showed that the independent predictor of PCa was only PSAD (Table 4). Moreover, ROC analysis revealed a cut-off value of PSAD as 0.11 in predicting PCa. With the cut-off value of 0.11 , the sensitivity and specificity were $70 \%$ and $71.1 \%$, respectively. Table 5 presents the number of patients with and without cancer and $p$-values when patients were divided based on the cut-off value of PSAD.

As regards the clinical significance of the PCa, PSAD was significantly higher and PV was significantly lower in patients with clinically significant $\mathrm{PCa}$ than in patients with clinically insignificant PCa, whereas PSA, free PSA, age and f/tPSA showed no significant difference between the two groups. AUCs were quite close to each other for PSAD and PV as 0.768 and 0.770 , respectively (Table 3 ).

These values which were found to be significant in a univariate analysis were compared by performing a multivariate analysis. The multivariate analysis revealed that only PSAD was an independent predictor for clinically significant PCa (Table 4). Moreover, ROC analysis revealed a cut-off value of PSAD as 0.199 in predicting clinically significant PCa. With the cut-off value of 0.199 , the sensitivity and specificity were $68.8 \%$ and $79 \%$, respectively. Table 5 presents the number of patients with and without clinically significant PCa and $p$-values when patients were divided based on the cut-off value of PSAD. 
Table 3. Comparison of patients' age, total PSA, free PSA, free to total PSA ratio, PSA density and prostate volume between clinically significant cancer and clinically insignificant cancer groups.

\begin{tabular}{|c|c|c|c|c|}
\hline \multirow[b]{2}{*}{ Parameters } & \multicolumn{2}{|c|}{$\begin{array}{r}\text { Clinical Significance } \\
n=40,100 \%\end{array}$} & \multirow[b]{2}{*}{$p$ value } & \multirow[b]{2}{*}{ AUC } \\
\hline & $\begin{array}{r}\text { Gleson score } \\
\leq 6 \\
n=24,60 \%\end{array}$ & $\begin{array}{r}\text { Gleson score } \\
\geq 7 \\
n=16,40 \%\end{array}$ & & \\
\hline $\begin{array}{r}\text { Age } \\
\text { Median (IQR) }\end{array}$ & $74.5(71-76)$ & $73 \quad(72-74.5)$ & 0.713 & 0.535 \\
\hline $\begin{array}{r}\text { Total PSA } \\
\text { Median (IQR) }\end{array}$ & $7.78(6.46-9.08)$ & $7.75(6.39-8.78)$ & 0.989 & 0.501 \\
\hline $\begin{array}{r}\text { Free PSA } \\
\text { Median (IQR) }\end{array}$ & $1.42(1.14-2.03)$ & $1.8(1.1-2.17)$ & 0.359 & 0.587 \\
\hline $\begin{array}{r}\text { Free/Total PSA ratio } \\
\text { Median (IQR) } \\
\end{array}$ & $0.20(0.14-0.27)$ & $0.24(0.18-0.33)$ & 0.233 & 0.615 \\
\hline $\begin{array}{r}\text { PSAD } \\
\text { Median (IQR) } \\
\end{array}$ & $0.12(0.08-0.19)$ & $0.28(0.13-0.34)$ & 0.004 & 0.768 \\
\hline $\begin{array}{r}\text { PV } \\
\text { Median (IQR) }\end{array}$ & $53 \quad(39-77.25)$ & $30 \quad(20-56)$ & 0.003 & 0.770 \\
\hline
\end{tabular}

PV: prostate volume, PSA: prostate specific antigen, PSAD: prostate specific antigen density, AUC: area under curve, IQR: interquartile range.

Table 4. Multivariate analysis of prostate volume, PSA density and free to total PSA ratio to determine independent predictors of PCa and clinically significant PCa.

\begin{tabular}{|r|r|r|r|r|r|r|}
\hline & \multicolumn{1}{|c|}{} & \multicolumn{2}{|c|}{ Prostate cancer } & \multicolumn{3}{|c|}{ Clinically significant prostate cancer } \\
\cline { 2 - 7 } & OR & \%95CI & P & OR & \%95CI & P \\
\hline PV & 1.018 & $0.995-1.041$ & 0.107 & 0.994 & $0.945-1.045$ & 0.809 \\
\hline PSAD & 0.001 & $0.000-0.002$ & $<0.001$ & 56292.21 & $\begin{array}{r}24797- \\
127790.545\end{array}$ & 0.006 \\
\hline f/tPSA & 3.007 & $0.085-106.025$ & 0.418 & - & - & - \\
\hline
\end{tabular}

PV: prostate volume, PSA: prostate specific antigen, PSAD: prostate specific antigen density, PCa: prostate cancer, f/t PSA: free/total PSA ratio, OR: odds ratio, $\mathrm{Cl}$ : confidence interval.

\section{DISCUSSION}

Uncertainties exist in cancer screening for older people. The clinical significance of prostate cancer screening is limited by a decreased life expectancy. However, in recent years, the elderly population has been found to have a longer life expectancy (6), and this indicates an increase in the number of elderly patients who may be candidates for prostate biopsy, especially in tertiary health institutions as ours to which elderly patients with lower urinary tract symptoms and/ or elevated PSA levels are referred. Nevertheless, increased PSA level alone may be an insufficient 
Table 5. The number of patients with and without prostate cancer and clinically significant prostate cancer and $p$ values when patients were separated according to determined cut-off values of PSAD.

\begin{tabular}{|c|c|c|c|}
\hline \multirow{2}{*}{ n (\%) } & & $\begin{array}{l}\text { PSA Density } \\
\mathrm{n}=130(100 \%)\end{array}$ & \multirow{2}{*}{$p$ value } \\
\hline & $\begin{array}{r}<0.11 \\
76(58.4 \%) \\
\end{array}$ & $\begin{array}{r}>0.11 \\
54(41.6 \%) \\
\end{array}$ & \\
\hline Cancer & 12 (15.8\%) & 28 (51.9\%) & \multirow[b]{2}{*}{$<0.001$} \\
\hline Non-cancer & 64 (84.2\%) & 26 (48.1\%) & \\
\hline \multirow{2}{*}{ n (\%) } & & $\begin{array}{l}\text { PSA Density } \\
n=40(100 \%)\end{array}$ & \\
\hline & $\begin{array}{r}<0.199 \\
24(60 \%) \\
\end{array}$ & $\begin{array}{r}>0.199 \\
16(40 \%) \\
\end{array}$ & $p$ value \\
\hline $\begin{array}{r}\text { Clinically Insignificant PCa } \\
\text { (Gleason score } \leq 6)\end{array}$ & 19 (79.1\%) & 5 (31.2\%) & \multirow{2}{*}{0.006} \\
\hline $\begin{array}{r}\text { Clinically Significant PCa } \\
\text { (Gleason score } \geq 7 \text { ) }\end{array}$ & 5 (20.9\%) & 11 (68.8\%) & \\
\hline
\end{tabular}

PSA: prostate specific antigen, PCa: prostate cancer.

parameter to make a biopsy decision in this elderly group of patients who are expected to have high age-related PSA. In this respect, Morgan et al. (8) clearly reported an age-related decline of PSA specificity. Similarly, in our study, no significant difference was noted between PSA levels of patients with and without cancer. Kobayashi et al. (9) and Yanai et al. (10) also highlighted the identical result for patients above 70 years. We are not doubtful on the use of PSA for PCa detection in older men, but other parameters that may help to predict PCa are needed. PSAD and f/tPSA have been studied extensively and PV is a relatively less studied parameter compared to the other two. To our knowledge, only three reports have focused on age and patients aged 70 years and older were examined in two of these studies (9-11).

In the current study, the cut-off value for PSAD in predicting patients with or without cancer was 0.11 . Stephan et al. (12) reported a similar value of 0.1 for patients with PSA between 4 and $10 \mathrm{ng} /$ $\mathrm{mL}$. These cut-off values are lower than those of most other studies which usually recommended 0.15 as the PSAD cut-off level (13-14). When these parameters were evaluated to predict clinically significant PCa, multivariate analysis again showed that only PSAD was an independent predictor and a cut-off value of 0.199 was determined for PSAD. In the literature, the recommended cut-off values for PSAD in predicting clinically significant PCa ranged from 0.08 to 0.32 , and in most of these studies, the proposed cut-off value was $0.15(11,13$, 14). On the other hand, only one of these studies focused on a specific age group. In this study, Kosaka et al. (11) reported that a cut-off value of 0.32 for PSAD was a very effective predictor of clinically significant PCa in men aged 50 years and younger. To the best of our knowledge, the PSAD cut-off value indicated in the present study is the only recommendation found in the literature on predicting clinically significant $\mathrm{PCa}$ in patients aged 70 years and older. PSAD $<0.15$, which is a suggested criteria of active surveillance in the European Association of Urology (EAU) guidelines in all age groups of PCa patients, can be readjusted for patients of different age groups, with the results of new studies targeting different age groups. In this way, evaluating patients with 
regard to active surveillance with the cut-off values adjusted for their age groups will yield healthier results. We emphasise that our recommendation is also important from this perspective. Although Yanai et al. (10) did not describe a cut-off value in predicting clinically significant PCa, the median PSAD value reported in patients with clinically significant PCa was 0.27 , consistent with ours (median, 0.28). However, in this study, clinically significant PCa was defined as the International Society of Urological Pathology (ISUP) grade 2 and above, whereas they defined it as ISUP grade 3 and above. Despite that, in predicting $\mathrm{PCa}$, they reported a PSAD cut-off value of 0.20 , which is higher than our recommendation (0.11) and many of the other studies (mostly around 0.15) $(13,14)$. Their median values were 0.20 for PSAD and $35.1 \mathrm{cc}$ for PV while ours were 0.101 and 71 $\mathrm{Cc}$, respectively. We associated the difference between the median values with the fact that they obtained prostate volumes using multiparametric magnetic resonance (mpMRI) which is considered to be a more accurate measurement tool compared to TRUS. Although it is necessary to underline that mpMRI may not be suitable for every patient in terms of time and cost, its use before prostate biopsy is becoming more common today. Wilson et al reported that PSAD and mpMRI were independent predictors of clinically significant PCa at biopsy (15). Furthermore, using PSAD combined with mpMRI has been reported to improve the negative predictive value of mpMRl, perform better than mpMRI alone in predicting clinically significant $\mathrm{PCa}$, and is also useful in making a biopsy decision in patients with inderterminate mpMRI lesions (16-18). Given the results of these studies and that mpMRI will be used more widely, we hope that the results we found about PSAD may be increased in importance by combining with mpMRI results.

Consistent with this study, Kobayashi et al. (9) reported that PSAD, PV and f/tPSA were significantly different for patients aged 70 years or older with and without cancer on univariate analysis. However, contrary to our study, they found on multivariate analysis that only PV was an independent predictor for PCa. In the present study, PV was not an independent predictor for PCa. Furthermore, Erdogan et al. (19) and Shigemura et al. (20) reported that PV was an independent predictor of PCa. However, any age classification was not performed in either study. Similar to the study by Kobayashi et al. (9) and the current one, Yanai et al. (10) reported that PV was significantly different between patients with and without cancer, including patients aged 70 years or older who also had a PSA level of $<20 \mathrm{ng} /$ $\mathrm{mL}$, but they did not make an evaluation for PV involving a multivariate analysis. When Erdoğan et al. (19) evaluated PV for clinically significant cancer, they did not achieve a meaningful result similar to our study. When all these data are evaluated as a whole, PV appears to be a significant parameter in predicting PCa but not for clinically significant PCa. Similar to our results, there are two studies showing that f/tPSA was significant in univariate analysis in predicting $\mathrm{PCa}$, but not in multivariate analysis. $(9,19)$. In one of these (9), contrary to our study, a significant difference for f/tPSA was found between patients with and without clinically significant cancer.

The limitations of our study included its relatively small sample size and retrospective nature. Although we aimed to minimise the errors due to sampling by including patients with at least 12-core biopsies, it is undeniable that in some patients, we may have missed PCa. Moreover, different biopsy criteria depending on patient age may cause selection biases to some degree. In this regard, we evaluated clinical parameters of biopsied patients in three age groups (70-75 years, 76-80 years and 81 years or older) and the cancer detection rates were not significantly different $(p=0.511)$. Other limitations can be considered as potential insufficiencies in the PV measurements due to TRUS measurements which 
may vary depending on the practitioner and in the Gleason score assessments due to the lack of radical prostatectomy specimens. However, all pathology results were reported by experienced pathologists since it was a tertiary hospital. The fact that our study is one of three studies conducted in patients aged 70 years and older and that the cutoff value of PSAD recommended for predicting clinically significant prostate cancers have not been investigated before for this age group are the strengths of our study.

In conclusion, the results of this study demonstrated that PSAD is an effective parameter in predicting $\mathrm{PCa}$ and clinically significant $\mathrm{PCa}$ in older patients. Moreover, it was superior to the other two parameters (PV and f/tPSA). We suggest that patients with a PSAD greater than 0.11 and 0.199 should be evaluated more carefully for $\mathrm{PCa}$ and clinically significant $\mathrm{PCa}$, respectively. Therefore, PSAD may play an active role in the decision-making for prostate biopsy in elderly men. Thereby, it was pointed out that unnecessary biopsies and possible complications can be decreased for elderly patients in accordance with

\section{REFERENCES}

1. Ferlay J, Soerjomataram I, Dikshit R, et al. Cancer incidence and mortality worldwide: sources, methods and major patterns in GLOBOCAN 2012. Int J Cancer 2015;136(5):E359-86. (PMID:25220842).

2. Siegel R, Ma J, Zou Z, Jemal A. Cancer statistics. CA Cancer J Clin 2014;64(1):9-29. (PMID:24399786).

3. Schröder F. Kattan MW. The comparability of models for predicting the risk of a positive prostate biopsy with prostate-specific antigen alone: a systematic review. Eur Urol 2008;54(2):274-90. (PMID:18511177).

4. Richie JP, Catalona WJ, Ahmann FR et al. Effect of patient age on early detection of prostate cancer with serum prostate-specific antigen and digital rectal examination. Urology 1993;42(4):365-74. (PMID:7692657).

5. Roobol MJ, Schröder FH, Hugosson J, et al. our recommendations. On the other hand, these recommendations should be confirmed with further studies.

\section{Disclaimers}

Author's statement that the views expressed in the submitted article are his or her own and not an official position of the institution or funder.

\section{Editorial \& Financial disclosure}

None of the contributing authors have any conflicts of interest, including editorial or financial interests and relationships and affiliations relevant to the subject matter or materials discussed in the manuscript. The authors declare that there is no conflict of interest regarding the publication of this paper.

\section{Funding support and role of the sponsor}

No funding or other financial support was.
Importance of prostate volume in the European Randomised Study of Screening for Prostate Cancer (ERSPC) risk calculators: results from the prostate biopsy collaborative group. World J Urol 2012;30(2):149-55. (PMID:22203238).

6. Siegel R, Miller KD, Jemal A. Cancer statistics. CA Cancer J Clin 2015:65(1):5-29. (PMID:25559415).

7. Gershman B, Van Houten HK, Herrin J, et al. Impact of prostate-specific antigen (PSA) screening trials and revised PSA screening guidelines on rates of prostate biopsy and postbiopsy complications. Eur Urol 2017;71(1):55-65. (PMID:26995328).

8. Morgan TO, Jacobsen SJ, McCarthy WF, Jacobson DJ, McLeod DG, Moul JW. Age-specific reference ranges for serum prostate-specific antigen in black men. $\mathrm{N}$ Engl J Med 1996;335(5):304-10. (PMID:8663870). 
9. Kobayashi T, Mitsumori K, Kawahara T, Nishizawa K, Ogura K, Ide Y. Prostate gland volume is a strong predictor of biopsy results in men 70 years or older with prostate? specific antigen levels of 2.0-10.0 ng/ mL. Int J Urol 2005;12(11):969-75. (PMID:16351653).

10. Yanai $Y$, Kosaka $T$, Hongo $H$, et al. Evaluation of prostate specific antigen density in the diagnosis of prostate cancer combined with magnetic resonance imaging before biopsy in men aged 70 years and older with elevated PSA. Mol Clin Oncol 2018;9(6):656-60. (PMID:30546897).

11. Kosaka $T$, Mizuno $R$, Shinojima $T$, et al. The implications of prostate-specific antigen density to predict clinically significant prostate cancer in men $\leq 50$ years. Am J Clin Exp Urol 2014;2(4):332-6. (PMID:25606579)

12. Stephan $C$, Stroebel $G$, Heinau $M$, et al. The ratio of prostate-specific antigen (PSA) to prostate volume (PSA density) as a parameter to improve the detection of prostate carcinoma in PSA values in the range of $<4 \mathrm{ng} / \mathrm{mL}$. Cancer 2005;104(5):993-1003. (PMID:16007682)

13. Ohori M, Dunn JK, Scardino PT. Is prostate-specific antigen density more useful than prostate-specific antigen levels in the diagnosis of prostate cancer? Urology 1995;46(5):666-71. (PMID:7495118).

14. Bazinet M, Meshref AW, Trudel C, et al. Prospective evaluation of prostate-specific antigen density and systematic biopsies for early detection of prostatic carcinoma. Urology 1994;43(6):44-51. (PMID:7506853).
15. Washino S, Okochi T, Saito K, et al. Combination of prostate imaging reporting and data system (PI-RADS) score and prostate-specific antigen (PSA) density predicts biopsy outcome in prostate biopsy naïve patients. BJU Int 2017;119(2):225-33. (PMID:26935594).

16. Distler FA, Radtke JP, Bonekamp D, et al. The Value of PSA Density in Combination with PI-RADS ${ }^{\text {TM }}$ for the Accuracy of Prostate Cancer Prediction. J Urol 2017;198(3):575-82. (PMID:28373135).

17. Han C, Liu S, Qin XB, Ma S, Zhu LN, Wang XY. MRI combined with PSA density in detecting clinically significant prostate cancer in patients with PSA serum levels of 4? $10 \mathrm{ng} / \mathrm{mL}$ : Biparametric versus multiparametric MRI. Diagn Interv Imaging 2020;101(4):235-44. (PMID:32063483).

18. Brizmohun Appayya M, Sidhu HS, Dikaios N, et al. Characterizing indeterminate (Likertscore 3/5) peripheral zone prostate lesions with PSA density, PI-RADS scoring and qualitative descriptors on multiparametric MRI. $\mathrm{Br} J$ Radiol 2018;91(1083):20170645. (PMID:29189042).

19. Erdogan A, Polat S, Keskin E, Turan A. Is prostate volume better than PSA density and free/total PSA ratio in predicting prostate cancer in patients with PSA $2.5-10 \mathrm{ng} / \mathrm{mL}$ and $10.1-30 \mathrm{ng} / \mathrm{mL}$ ?. Aging Male 2020;23(1):59-65. (PMID:30862227).

20. Shigemura K, Arakawa S, Yamanaka K, et al. Potential predictive factors of positive prostate biopsy in the Japanese population. Int Urol Nephrol 2008;40(1):916. (PMID:17610138). 